\title{
Performance Analysis of CAR: Centralized Adaptive Reservation*
}

\author{
Henry C. B. Chan', Victor O. K. Li ${ }^{2}$ and Victor C. M. Leung ${ }^{3}$ \\ ${ }^{1}$ Department of Computing, Hong Kong Polytechnic University, Hong Kong \\ ${ }^{2}$ Department of Electrical \& Electronic Engineering, University of Hong Kong, Hong Kong \\ ${ }^{3}$ Department of Electrical and Computer Engineering, University of British Columbia, Canada
}

\begin{abstract}
A BSTRACT
A centralized adaptive reservation (CAR) protocol has been proposed in [1] for supporting constant bit rate (CBR), variable bit rate (VBR) and unspecified bit rate (UBR) services in a starconfigured network. This protocol has two main features. First, both the access probability and the bandwidth for reservation requests are varied dynamically to enhance efficiency. Second, by using a request-mini-slot method, UBR traffic can only utilize the residual bandwidth of the other traffic. In this paper, we derive a Markov chain model for analyzing the performance of the CAR protocol by means of a bi-state traffic source. The focus is to analyze the performance of the VBR stations when both the access probability and the bandwidth for reservation requests are changed dynamically.
\end{abstract}

\section{INTRODUCTION}

Many communication networks [2-6] are based on a star topology in which distributed stations share a common communication channel managed by a centralized controller (CC), typically under a time-division multiplexing system. Packet reservation protocols are well suited for providing medium access control for this type of network. In [1], a centralized adaptive reservation (CAR) protocol has been proposed for integrating constant bit rate (CBR) traffic, variable bit rate (VBR) traffic and unspecified bit rate (UBR) traffic over star-configured networks. Compared to previous packet reservation protocols, such as $[2,4,5,7,8,9]$, the CAR protocol enhances efficiency by dynamically varying both the access probability and the bandwidth for reservation requests. Furthermore, by means of a request-minislot method, it is guaranteed that the UBR traffic can only utilize the residual bandwidth of the CBR and VBR traffic.

A key research problem on the CAR protocol is to investigate how a VBR station can connect to the CC efficiently when it becomes active again. In this paper, we derive a Markov chain model for analyzing the performance of the CAR protocol by means of a bi-state traffic source. Functioning as a mini-source, this bi-state traffic source can also be used as a building block for general VBR traffic sources. We use the model to evaluate the packet loss ratio when the access probability is varied according to the dynamic p-persistent protocol in [1]. The results are compared with those of the ideal case, in which the CC knows how many stations are contending for the reservation mini-slots. The results indicate that the CAR protocol can yield performance close to the ideal case. Using the model, we also evaluate the residual bandwidth available for the UBR traffic. The analysis gives us a better understanding of the CAR protocol and provides valuable insights into the design of packet reservation protocols in general.

The rest of the paper is organized as follows. Section 2 presents an analytical model for evaluating the performance of the CAR protocol. Section 3 presents and discusses the analytical results. Section 4 gives the conclusions.

\section{PERFORMANCE MODEL}

Let us first give an overview of the CAR protocol [1]. The channel is framed and slotted, with each slot carrying an ATM cell. An idle slot can also be divided into 6 mini-slots for carrying reservation requests. For $C B R$ traffic, the required number of slots per frame is pre-assigned during call setup. For VBR traffic, slots are assigned dynamically. Basically, when a VBR station starts an active period, it first acquires a mini-slot using the dynamic p-persistent protocol [1] to convey the reservation request. During the active period, the VBR station may also change its bit rate by piggybacking the new bit rate requirement. UBR stations also connect to the $\mathrm{CC}$ through random access and then piggyback subsequent slot requirements. A request-mini-slot method is used to detect whether there are capturing VBR stations in the network, so that UBR traffic can be disabled accordingly.

We assume that there are $F$ slots per frame and the propagation delay is negligible. There are $N_{v}$ voice stations, each alternating independently between active and idle states. In general, these voice stations can be used as mini-sources for modeling VBR traffic. The active and idle periods are independent and exponentially distributed with means of $\frac{l}{\beta}$ secs. and $\frac{l}{\alpha}$ secs., respectively, for an activity factor of $\phi=\frac{\alpha}{\alpha+\beta}$. If a voice station is in idle state at frame $k$, the probability that it will be in active state at frame $k+1$ is $q_{i a}=1-e^{-\alpha f}$ where $f$ is the frame duration (6 $\mathrm{ms}$ in this paper). If a voice station is active in frame $k$, the probability that it will be idle in frame $k+1$ is

*This work was supported by a Hong Kong Polytechnic University Research Grant under account number: A-PB23. 
$q_{a i}=I-e^{-\beta f}$. State transitions only occur at the frame boundaries. During the active state, a voice station requires one reserved slot per frame for its packet transmission. Due to the use of silent packet suppression, no slot is required during the idle state and the previously reserved slot can be released. When an idle voice station becomes active, it first accesses a mini-slot (through random access) for making the reservation request to the $\mathrm{CC}$. Once it successfully accesses a mini-slot in frame $k$, the first packet transmission occurs in frame $k+1$ and isochronous service can be provided for its subsequent packet transmissions. If a capturing station cannot acquire a mini-slot in frame $k$, a packet will be dropped in frame $k+1$ because no reserved slot is available for the packet transmission.

At the beginning of frame $k$, there are $c_{k}$ capturing voice stations (active voice stations which need to access a mini-slot to convey the reservation requests to the $\mathrm{CC}$ ), $a_{k}$ active voice stations and $r_{k}$ reserved voice stations (those with successful reservations at the $\mathrm{CC}$ ). In frame $k$, there are $m_{k}$ mini-slots for serving the reservation requests and $r_{k}$ reserved slots for the reserved voice stations.

Each capturing voice station accesses a mini-slot with probability $p_{k}$. If a mini-slot is accessed by more than one station, a collision occurs and the mini-slot is wasted. If a minislot is accessed by only one station, the reservation request can be conveyed to the CC. For simplicity, immediate feedback and error-free transmissions are assumed. For a successful reservation request, the corresponding station does not need to access a minislot again. For non-successful reservation requests (i.e., collisions), the respective stations need to access the next minislot with probability $p_{k}$ again. For each successful reservation request received in frame $k$, a slot (if available) will be reserved for the respective voice station in frame $k+1$. The number of minislots in frame $k+1$ will then be decreased accordingly. It is possible that the available slots are insufficient to serve all successful reservation requests. This can only occur when $r_{k+l}=F$, otherwise all the reservation requests must be fulfilled. In this case, the affected stations need to fall back to the capture state in the next frame to access a mini-slot again. Note that in reality, these unsuccessful requests can still be registered by the $\mathrm{CC}$ so that they can be served later when slots are available. To facilitate the following analysis, the "fall back" assumption is required.

An embedded Markov chain is formed with the embedded point at the beginning of each frame. The state of the Markov chain is defined by two variables: $\left\{c_{k}, r_{k}\right\}$. This Markov chain is ergodic because it has a finite number of states and is obviously irreducible. Therefore, the stationary probability for each state exists. Denote $y_{k}$ as the number of stations which have successfully accessed a mini-slot in frame $k, z_{k}$ as the number of reserved stations which have the last packet transmissions in frame $k$ and will therefore release the reserved slots in frame $k+1$, $v_{k}$ as the number of stations which had successfully accessed a mini-slot in frame $k$ but cannot find a reserved slot at the end of frame $k$ (i.e., there are insufficient slots for the case $r_{k+1}=F$ ), and $w_{k}$ as the number of capturing stations which returns to the idle state at the end of frame $k$ (i.e., it no longer needs to access a mini-slot in frame $k+1$ ). Referring to the state transition diagram in Fig. 1, we can set up the following equations:

$$
\begin{aligned}
& r_{k+1}=\min \left(F, r_{k}+y_{k}-z_{k}\right) \\
& v_{k}=\left\{\begin{array}{c}
r_{k}+y_{k}-z_{k}-F \quad r_{k+1}=F \\
0 \quad \text { otherwise }
\end{array}\right. \\
& c_{k+1}=c_{k}+\left(v_{k}-y_{k}\right)+\left(x_{k}-w_{k}\right)=c_{k}+\left(v_{k}-y_{k}\right)+u_{k}
\end{aligned}
$$

where $u_{k}=\left(x_{k}-w_{k}\right)$

We first consider the case where $v_{k}=0$ or equivalently $r_{k+1} \neq F$. It is not difficult to set up the following inequalities: $0 \leq x_{k} \leq N_{v}-r_{k}-c_{k}, 0 \leq y_{k} \leq c_{k}$, $0 \leq z_{k} \leq r_{k}$ and $0 \leq w_{k} \leq c_{k}-y_{k}$

Based on the above, we can also set up the following inequality for $u_{k}: y_{k}-c_{k} \leq u_{k} \leq N_{v}-r_{k}-c_{k}$

From (1)-(3), it can be seen that given $\left\{c_{k}, r_{k}\right\}$ and $\left\{c_{k+1}, r_{k+1}\right\}, y_{k}$ and $u_{k}$ can be expressed in terms of $z_{k}$ as follows: $y_{k}=r_{k+1}-r_{k}+z_{k} \quad$ and $u_{k}=c_{k+1}-c_{k}+r_{k+1}-r_{k}+z_{k}$. Denote $\zeta$ as the indicator function which indicates that the above inequalities are satisfied, i.e.,

$\zeta= \begin{cases}I & 0 \leq y_{k} \leq c_{k}, y_{k}-c_{k} \leq u_{k} \leq N_{v}-r_{k}-c_{k} \\ 0 & \text { otherwise }\end{cases}$

The transition probability of the Markov chain can be found by summing over all the possible $\bar{z}_{k}$ as shown in the following equation:

$$
\begin{aligned}
& P\left(c_{k+1}, r_{k+1} \mid c_{k}, r_{k}\right) \\
& =\sum_{z_{k=0}}^{r_{k}} P_{R I}\left(z_{k} \mid r_{k}\right) \times P_{C R}\left(v_{k} \mid c_{k}, m_{k}\right) \times P_{U}\left(u_{k} \mid c_{k}, r_{k}\right) \times \zeta \\
& =H_{l}\left(c_{k+1}, r_{k+1}, c_{k}, r_{k}\right)
\end{aligned}
$$

where $P_{R I}\left(z_{k} \mid r_{k}\right)$ is the probability that $z_{k}$ stations leave the reserved state given that there are currently $r_{k}$ reserved stations, $P_{C R}\left(y_{k} \mid c_{k}, m_{k}\right)$ is the probability that $y_{k}$ stations captures a mini-slot successfully given that there are $c_{k}$ capturing stations and $m_{k}$ mini-slots in frame $k$, and $P_{U}\left(u_{k} \mid c_{k}, r_{k}\right)$ is the probability that a net number of $u_{k}$ stations enters the capture state given that there are $c_{k}$ capturing stations and $r_{k}$ reserved stations. Obviously $P_{R I}\left(z_{k} \mid r_{k}\right)$ can be found easily by the following equation:

$$
P_{R I}\left(z_{k} \mid r_{k}\right)=\left(\begin{array}{l}
r_{k} \\
z_{k}
\end{array}\right) q_{a i}{ }^{z_{k}}\left(I-q_{a i}\right)^{r_{k}-z_{k}}=B\left(r_{k}, z_{k}, q_{a i}\right)
$$

where 
$B(n, h, l)=\left\{\begin{array}{l}\left(\begin{array}{l}n \\ h\end{array}\right) l^{h}(l-l)^{n-h} \quad n \geq h, h \geq 0 \\ 0 \quad \text { otherwise }\end{array}\right.$

$P_{C R}\left(y_{k} \mid c_{k}, m_{k}\right)$ can be found recursively by using the following equation:

$$
\begin{aligned}
& P_{C R}\left(y_{k} \mid c_{k}, m_{k}\right) \\
& = \begin{cases}G_{l}\left(y_{k}, c_{k}, m_{k}\right)+G_{2}\left(y_{k}, c_{k}, m_{k}\right) \quad y_{k} \leq \min \left(c_{k}, m_{k}\right) \\
l \quad y_{k}=0, c_{k}=0 \text { or } y_{k}=0, m_{k}=0 \\
0 \quad \text { otherwise }\end{cases}
\end{aligned}
$$

where

$$
\begin{aligned}
& G_{l}\left(y_{k}, c_{k}, m_{k}\right) \\
& \quad=P_{C R}\left(y_{k}-I \mid c_{k}, m_{k}-l\right)\left(c_{k}-y_{k}+1\right) p_{k}\left(1-p_{k}\right)^{c_{k}-y_{k}}
\end{aligned}
$$

$$
\begin{aligned}
& G_{2}\left(y_{k}, c_{k}, m_{k}\right) \\
& \quad=P_{C R}\left(y_{k} \mid c_{k}, m_{k}-I\right)\left(I-\left(c_{k}-y_{k}\right) p_{k}\left(I-p_{k}\right)^{c_{k}-y_{k}-l}\right)
\end{aligned}
$$

Let us explain these equations in details. Suppose that there are $c_{k}$ capturing stations at the beginning of frame $k$. If $y_{k}$ stations captures a mini-slot successfully just after the $m_{k}$-th minislot, there are only two possible situations. In the first situation, as given by (9), there are $y_{k}-1$ successful stations just after the $\left(m_{k}-I\right)$-th mini-slot and one station captures the $m_{k}$-th minislot. In the second situation as given by $(10)$, there are $y_{k}$ successful stations just after the $\left(m_{k}-l\right)$-th mini-slot and no station captures the $m_{k}$-th mini-slot. Hence the first case of $(8)$ is as shown above. Of course, if either $c_{k}$ or $m_{k}$ is zero, $y_{k}$ must be zero. This gives the second case of (8). The recursive equation can be started by considering the situation at the first mini-slot as follows:

$$
\begin{aligned}
& P_{C R}\left(0 \mid c_{k}, l\right)=l-c_{k} p_{k}(l-p)^{c_{k}-l} \\
& P_{C R}\left(l \mid c_{k}, I\right)=c_{k} p_{k}\left(l-p_{k}\right)^{c_{k}-l} \\
& \quad P_{U}\left(u_{k} \mid c_{k}, r_{k}\right) \text { can be found from the following equation } \\
& N_{v}-c_{k}-r_{k} \\
& \quad \sum_{k}=0 \\
& =P_{U}\left(u_{k} \mid c_{k}, r_{k}\right)
\end{aligned}
$$

Recall that in each of the above binomial distribution expressions, the value is zero if the second term is zero or larger than the first term. Also, in the current case, the value of $v_{k}$ is zero.

When $r_{k+1}=F$, it is possible that some stations which have captured a mini-slot successfully may need to fall back to the capturing state. In this case, the transition probability becomes

$$
P\left(c_{k+1}, r_{k+1} \mid{ }_{k}, r_{k}\right)
$$

$=\sum_{z_{k}=0 y_{k}=F-r_{k}+z_{k}}^{r_{k}} P_{R I}^{c_{k}}\left(z_{k} \mid r_{k}\right) \times P_{C R}\left(y_{k} \mid c_{k}, m_{k}\right) \times P_{U}\left(u_{k} \mid c_{k}, r_{k}\right) \times \zeta$

$=H_{2}\left(c_{k+l}, r_{k+l}, c_{k}, r_{k}\right)$

where $u_{k}=c_{k+1}-c_{k}+y_{k}-v_{k}=c_{k+I}-c_{k}-r_{k}+z_{k}+F$

Basically, $y_{k}$ can now take values from $y_{k}=r_{k+1}-r_{k}+z_{k}=F-r_{k}+z_{k}$ to $c_{k}$. The number of unfulfilled capture requests is $v_{k}=r_{k}+y_{k}-z_{k}-F$.

Putting the above results together, the transition probability can be expressed as:

$P\left(c_{k+1}, r_{k+1} \mid c_{k}, r_{k}\right)$
$=\left\{\begin{array}{cc}H_{1}\left(c_{k+1}, r_{k+1}, c_{k}, r_{k}\right) & r_{k+1}<F \\ H_{2}\left(c_{k+1}, r_{k+1}, c_{k}, r_{k}\right) & r_{k+1}=F \\ 0 & \text { otherwise }\end{array}\right.$

Having found the transition probability of the Markov chain, we can find the stationary probabilities $\pi_{c, r} \quad\left(0 \leq c \leq N_{v}\right.$, $0 \leq r \leq \min \left(F, N_{v}-c\right)$ ). Having determined $\pi_{c, r}$, we can evaluate a number of interested performance indicators. The first one is the packet loss ratio (PLR) $P_{\text {loss }}$ which is defined as the fraction of packets being discarded. If the state at the beginning of a frame is $\{c, r\}$ (i.e., $c$ capturing stations and $r$ reserved stations) which occurs with a probability $\pi_{c, r}$ and there are $m$ mini-slots available for the reservation request, the packet loss ratio can be found as follows.

$P_{\text {loss }}=$

$$
\left.\frac{1}{\phi N_{v}} \sum_{c=0}^{N_{v}} \sum_{r=0}^{\left.m i \infty F N_{p}-c\right)} \sum_{i=0 j=0}^{r} \sum_{j=0}^{c}(c-j+(j-\sigma-r+i))^{\dagger}\right) B\left(r, i, q_{a i}\right)_{C R}^{P(j k, m) \pi}{ }_{c}, r
$$

Let us explain the above equation in detail. Suppose that $i$ of the $r$ reserved stations end the active state (i.e., transmits the last packets in the current frame and releases the slots in the next frame) and $j$ of the capturing stations capture a mini-slot each. The former and latter situations occur independently with probabilities $B\left(r, i, q_{a i}\right)$ and $P_{C R}(j \mid c, m)$, respectively. Therefore at least $c-j$ packets are dropped in the next frame because they do not have a reserved slot. Now, the number of slots available in the next frame is give by $(F-r+i)$ because of the departure of the $i$ reserved stations. Hence, $(j-(F-r+i))$ stations which have captured mini-slots successfully also need to fall back to the capture state. Consequently, the total number of packets dropped in the next frame is $\left(c-j+(j-(F-r+i))^{+}\right)$. Summing over all the possible $i, j, r$ and $c$, the packet loss ratio can be found by (16) noting that the denominator gives the average number of packets transmitted per frame.

Slot utilization, which is defined as the percentage of slots being utilized to carry VBR traffic, can be found as follows: 


$$
U=\frac{100}{F} \sum_{c=0}^{N_{v}} \sum_{r=0}^{\min \left(F, N_{v}-c\right)} r \pi_{c, r}
$$

In the CAR protocol, UBR traffic can only use the channel when there is no capturing voice station at the beginning of a slot. Unfortunately, this is difficult to evaluate. In this model, we assume that UBR traffic can only utilize the channel when there is no capturing voice station at the beginning of a frame, which is in fact a more stringent requirement (i.e., the actual available bandwidth is higher). The bandwidth utilization for the UBR traffic is defined as the percentage of bandwidth available for the UBR traffic as follows:

$$
U_{\text {residual }}=\frac{100}{F} \sum_{r=0}^{N_{v}}(F-r) \pi_{\ell, r}
$$

Note that ideally the bandwidth utilization for the UBR traffic should be $\frac{10 O}{F}\left(F-\phi N_{v}\right)$, where $\phi N_{v}$, gives the average number of slots per frame used by the voice stations. It is of interest to compare (18) with this ideal result.

\section{ANALYTICAL RESULTS AND DISCUSSION}

In this section, we employ the above model to analyze the performance of the CAR protocol. Our main focus is to evaluate the performance when both the access probability and the bandwidth for reservation request are changed dynamically. Unless otherwise specified, we assume that $F=10, \frac{1}{\beta}=1.5 \mathrm{secs}$. and $\frac{l}{\alpha}=2.25$ secs.

We first consider that a fixed number of mini-slots is allocated and the access probability can either be fixed or varied dynamically (dynamic) according to [1]. For the fixed access probability case, we use $p_{k}=0.2$ based on the access probabilities used by most packet reservation protocols. Fig. 2 shows the results for the case $m_{k}=m=6$ (i.e., 6 mini-slots are assigned for reservation request). For comparison, we also evaluate the PLR for the case where the bandwidth for reservation request is varied dynamically by turning all unreserved slots to mini-slots $m_{k}=6\left(F-r_{k}\right)$ and the access probability in each

frame is set to $p_{k}=\frac{1}{c_{k}}$. We refer to this as the ideal case ${ }^{1}$. It can be seen from Fig. 2 that if the access probability is varied dynamically, the PLR becomes smaller as compared to the result for fixed access probability. However, it is still much larger than the ideal result, especially when the number of stations is small (or the target PLR is small). Fig. 3 shows the result when the number of fixed mini-slots per frame is increased to 12. There are some differences with the previous result. When the number of stations is large, the PLR for dynamic access probability is lower than that for fixed access probability. This indicates that when

${ }^{1}$ Strictly speaking, this is not the ideal case because the number of capturing stations changes continuously within a frame and the access probability should also be changed on a per mini-slot basis. For practical implementation, we consider that the access probability only changes at the beginning of each frame and refer to this as the ideal case. more mini-slots are available, a lower PLR can be achieved by varying the access probability dynamically. In this case, the performance is close to that achieved by the ideal case. However, as the number of stations decreases (i.e., fewer stations contend for the mini-slots), the PLR for dynamic access probability and that for fixed access probability are almost the same. In this case, the performance is much poorer than the ideal performance.

We now consider that the bandwidth for reservation request is varied dynamically but the access probability can either be fixed $p=0.2$ ) or varied dynamically. Again we compare the results with the ideal result (ideal). Fig. 4 shows that the PLR for the case of dynamic access probability is almost the same as the ideal result. When the number of stations is large, however, all three cases show similar performance. This indicates that it is better to use a dynamic access probability if the required PLR is low (i.e., quality of service is high). Although, with a high PLR target, it is still possible to achieve performance close to the ideal result by using an appropriate fixed access probability, it may be difficult to determine its value. Setting the probability too low will result too many idle slots but setting it too high will cause too many contentions. Hence it is still preferable to use the dynamic access probability in general. Fig. 5 shows the PLR for the three cases when $F$ is changed while keeping $N_{\mathrm{r}}$, at $[1.2 F\rfloor$.

It further illustrates that the PLR for the case of dynamic access probability is very close to the ideal result and the performance improvement is more significant when the PLR target is low.

Fig. 6 shows that in terms of slot utilization, all three cases have almost the same result. This is because of the reservation nature of the protocol. Although the initial access is subject to access contention and hence slot wastage, it only accounts for a small part of the talkspurt. Once a reservation is secured, there will be no contention for all three cases, thus resulting in almost the same slot utilization.

It is also of interest to vary the active and idle periods while keeping the same activity factor $\phi$. This is done by increasing/ decreasing the active and idle periods with the same scaling factor. Fig. 7 shows the packet loss ratio for $N_{v}=17$ when the active period is varied. It shows that unless the active period is close to the frame duration, the result for dynamic access probability is close to the ideal performance. As expected, when the active period moves closer to the frame period, the PLR rises sharply because stations change state very frequently, thus resulting in more access contentions.

Fig. 8 shows the residual bandwidth available for the UBR traffic as found by (18). Recall that because of the use of the request-mini-slot method, UBR traffic can only access the channel when there is no capturing station at the beginning of a frame (i.e., $c=0$ ). Results indicate that the residual bandwidth is very close to the ideal result as evaluated by $\frac{I 0 f}{F}\left(F-\phi N_{v}\right)$. This indicates that it is justifiable to resolve the access contention of the voice stations first instead of allowing the UBR stations to contend for bandwidth with the voice stations (or VBR stations in general). This method can also be extended to provide priority control by introducing a number of request-mini-slots to ensure that lower priority traffic can only utilize the channel when higher priority traffic has resolved its access contentions. On-going 
research is being conducted to investigate this method together with the adaptive reservation protocol.

\section{CONCLUSIONS}

In conclusion, the proposed CAR protocol can be used for integrating CBR, VBR and UBR traffic over a star-configured network under a time-division multiplexing system. A key feature of the CAR protocol is that both the access probability and the bandwidth for reservation request are changed dynamically to enhance performance. Furthermore, it is guaranteed that the UBR traffic can only be transmitted over the residual bandwidth of the CBR and VBR traffic. We have derived a Markov chain model for analyzing the performance of the CAR protocol by means of a bi-state traffic model. Our focus is to analyze the performance when both the access probability and bandwidth for reservation request are varied dynamically. The model can also be extended to analyze general VBR traffic. The analytical results give us a better understanding of the protocol and provide valuable insights into the design of the CAR protocol in general. Results indicate that by varying both access probability and bandwidth for reservation request, the CAR protocol can yield performance close to the ideal case as defined earlier. The performance improvement is more significant when the required quality of service is higher. Consequently, by combining the advantages of centralized control and distributed access, the CAR protocol presents an effective "vehicle" to deliver CBR, VBR and UBR services for a star-configured communications network. Further work is being conducted to refine the protocol and the performance model.

\section{REFERENCES}

[1] H.C.B. Chan and V.C.M. Leung, "Centralized Adaptive Reservation (CAR) for Star-Configured Networks", in Proc. IEEE CCECE' 00 , Halifax, NS, May 2000.

[2] D. J. Goodman, R. A. Valenzuela, K. T. Gayliard and B. Ramamurthi, "Packet reservation multiple access for local wireless communications", IEEE Trans. on Commun., vol. 37, pp. 885-890, Aug. 89.

[3] D. Sala and J. O. Limb, "A protocol for efficient transfer of data over fiber/cable systems", in Proc. Infocom '96, San Francisco, pp. 904-911, Mar. 1996.

[4] V. C. M. Leung, "Packet reservation protocols for multichannel satellite networks", IEE Proceedings-I, vol. 140, No. 6, pp.453-461, Dec. 1993.

[5] N. Amitay and S. Nanda, "Resource auction multiple access for statistical multiplexing of speech in wireless PCS", IEEE Trans. Veh. Tech., vol. 43, no. 3, pp.584-596, Aug. 1994.

[6] D. Raychaudhuri et al., "WATMnet: A Prototype Wireless ATM System for Multimedia Personal Communication", IEEE J. Selec. Area in Commun, vol. 15, no.1, Jan 1997, pp. 83-95.
[7] J. H. Wen and J. W. Wang, "A non-collision PRMA protocol for integrated voice and data wireless networks", in Proc. ICUPC'95, pp. 462-466, 1995.

[8] G. Bianchi, F. Borgonovo, L. Fratra, L. Musumeci and M. Zurzi, "C-PRMA: the centralized packet reservation multiple access for local wireless communications", in Proc. Globecom'94, San Francisco, USA, pp. 1340-1346, Nov. 1994.

[9] X. Qiu and V. O. K. Li, "Dynamic reservation multiple access (DRMA): A new multiple access scheme for personal communication system", ACM/Baltzer Wireless Networks, vol. 2, pp. 117-128, 1996.

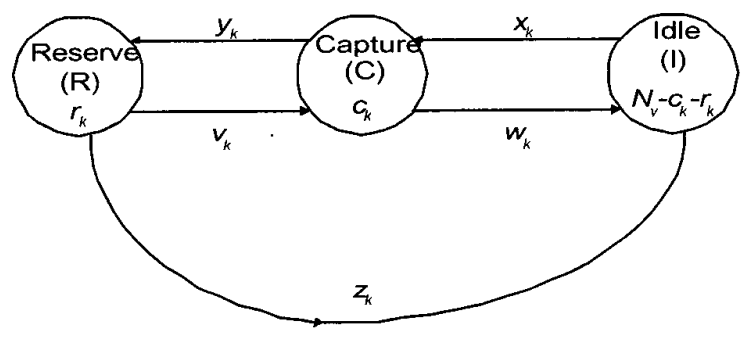

Figure 1: State diagram

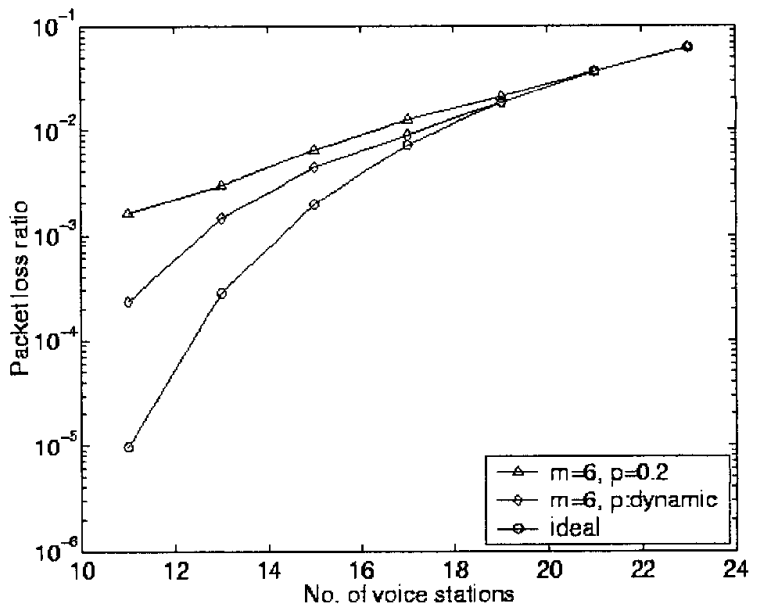

Figure 2: PLR when $m=6$ (fixed no. of mini-slots) 


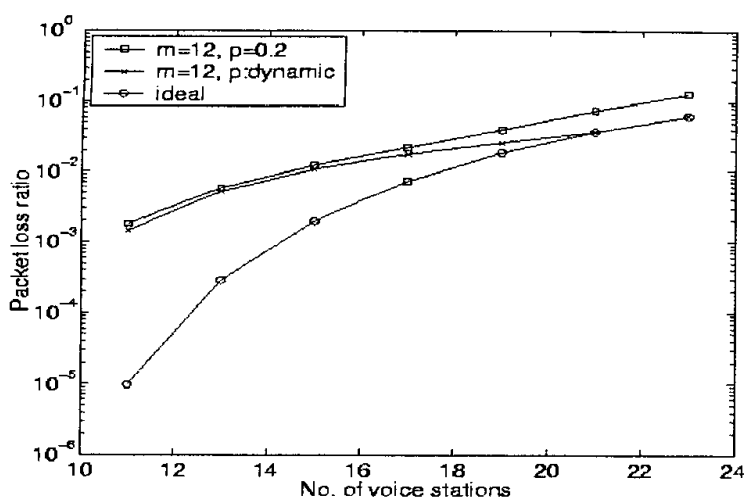

Figure 3: PLR when $m=12$ (fixed no. of mini-slots)

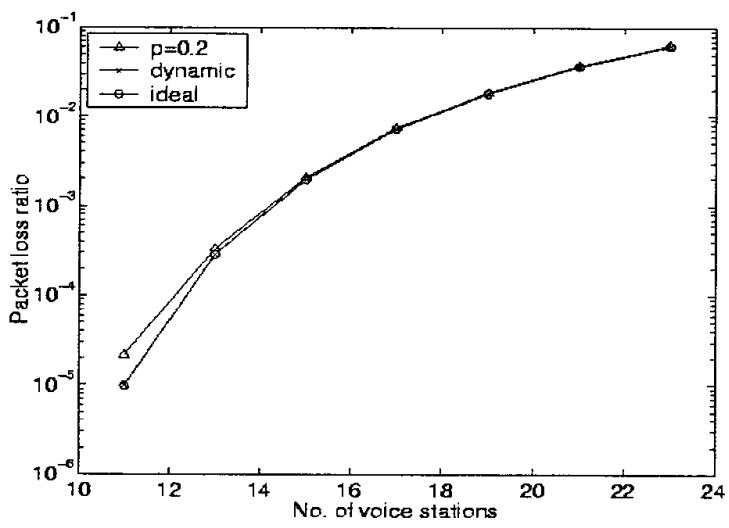

Figure 4: PLR when $m$ is varied dynamicallyl

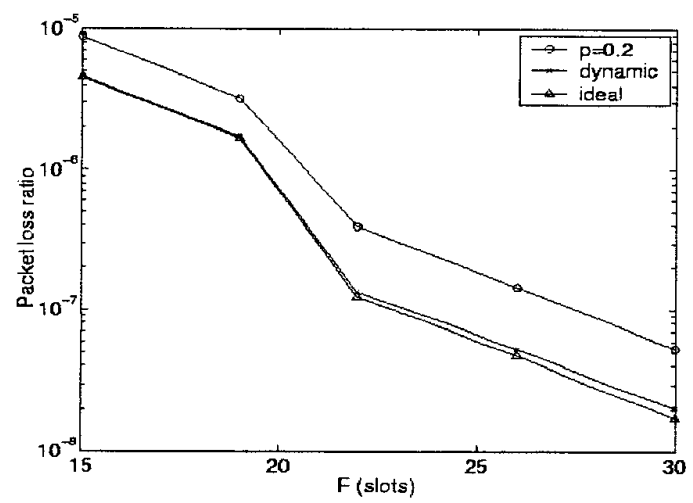

Figure 5: PLR for different $F$ when $m$ is varied dynamically

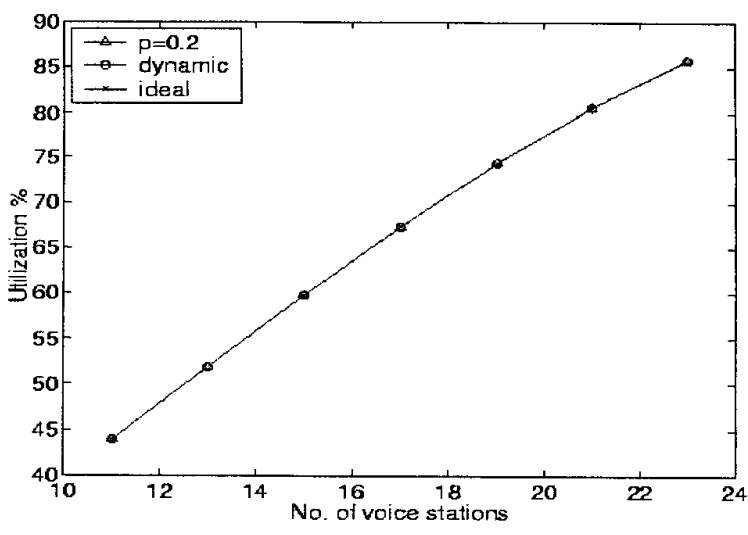

Figure 6: Slot utilization

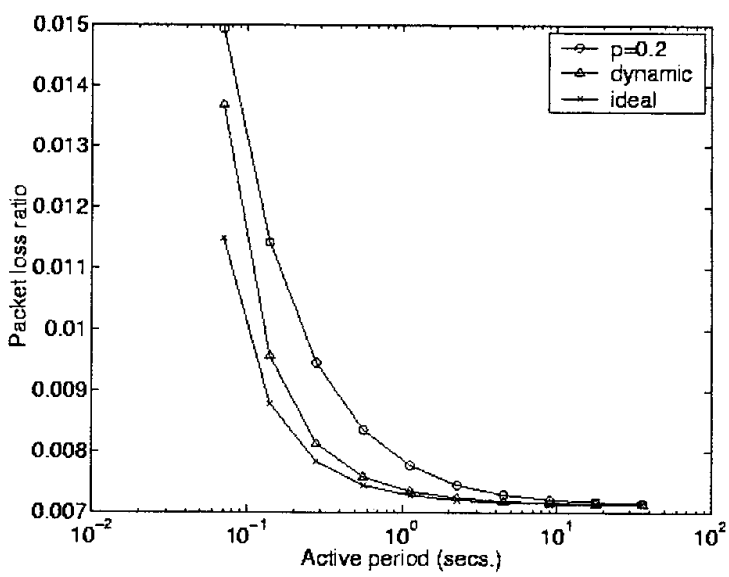

Figure 7: PLR when active/idle period is varied while keeping the same activity factor

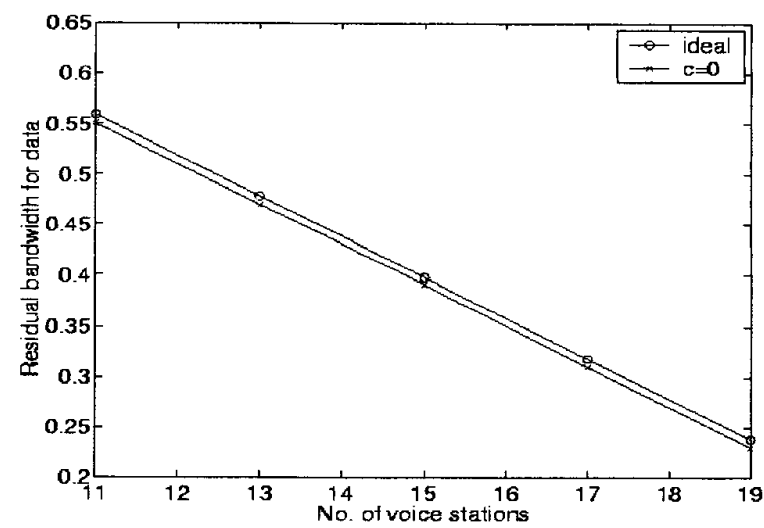

Figure 8: Residual bandwidth available for UBR traffic 